\title{
Title
}

\section{A distinctive neural nexus predicts Braille reading proficiency in blind individuals}

\section{Authors}

Ruxue Wang ${ }^{1, \dagger}$, Jiangtao Gong ${ }^{2,3, \dagger}$, Chenying Zhao ${ }^{1}$, Yingqing $\mathrm{Xu}^{2,3, *}$ and Bo Hong ${ }^{1,4, *}$

${ }^{1}$ Department of Biomedical Engineering, School of Medicine, Tsinghua University, Beijing, 100084, P.R. China;

${ }^{2}$ The Future Laboratory, Tsinghua University, Beijing, 100084, P.R. China;

${ }^{3}$ Academy of Arts \& Design, Tsinghua University, Beijing, 100084, P.R. China;

${ }^{4}$ IDG/McGovern Institute for Brain Research, Tsinghua University, Beijing, 100084, P.R. China

'R.W. and J. G. contributed equally to this work.

*Correspondence and requests for materials should be addressed to Bo Hong (email: hongbo@tsinghua.edu.cn) or to YingqingXu (email: yqxu@tsinghua.edu.cn). 


\begin{abstract}
In the absence of visual input, the occipital 'visual' cortex of blind people takes on nonvisual cognitive functions. Although the increased functional connectivity between 'visual' cortex and frontal cortex in the blind has been observed, to what extent this connectivity contributes to cognitive behaviour in blind individuals remains unclear. Here, we found enhanced functional connectivity between higher-tier 'visual' cortexthe lateral occipital cortex (LOC), but not the primary 'visual' cortex, and typical frontal language areas - the inferior frontal cortex (IFC) in people with acquired blindness. Blind individuals' Braille reading proficiency is predicted positively by the leftlateralized LOC-IFC connectivity. Further, an increased bidirectional information flow between the left LOC and IFC was observed during a natural Braille reading task. In particular, the task modulation of the top-down communication from left IFC to LOC was significantly stronger than that of the bottom-up one. Our study revealed a distinctive neural nexus, left LOC-IFC connection, and its behavioural significance for natural Braille reading in acquired blindness, providing new evidence for the crossmodal plasticity in human cortex.
\end{abstract}




\section{Introduction}

Tactile Braille reading is a demanding skill that allows blind humans to achieve literacy (Sadato et al., 1998; Sadato, 2005). Beyond the somatosensory tactile processing system, it has been shown that Braille words significantly activate an extended area of the 'visual' cortex stretching from the primary 'visual' cortex (V1) to higher-tier 'visual' regions - the lateral occipital cortex (LOC) in blind humans (Hamilton et al., 2000; Burton, Snyder, Conturo, et al., 2002; Burton, 2003; Pascual-Leone et al., 2005; Reich et al., 2011), including peak activation in the approximately consistent area as the typical visual word form area (VWFA) in sighted individuals (Reich et al., 2011; Kim et al., 2017; Katarzyna Rączy et al., 2019). The recruitment of the higher-order language system has also been observed (Burton, Snyder, Conturo, et al., 2002; Kim et al., 2017; Katarzyna Rączy et al., 2019). However, while there are several studies on the neural correlates of Braille reading, most of these studies employed either only Braille letter (Cohen et al., 1997; Fujii et al., 2009; Bola et al., 2019) or Braille word (Sadato et al., 1998; Burton, Snyder, Conturo, et al., 2002; Amedi et al., 2003; Reich et al., 2011; Siuda-Krzywicka et al., 2016; Kim et al., 2017; Katarzyna Rączy et al., 2019; Matuszewski et al., 2021) recognition rather than the natural Braille reading of complete sentences. Therefore, the neural basis of natural Braille reading, especially the interaction of the visual system and genuine language system, is not yet fully understood.

Moreover, functional connectivity patterns between the 'visual' cortex and other areas of the brain have been found to be altered in blind humans. One of the most robustly found changes in connectivity is that between the 'visual' cortex and frontal cortex (Liu et al., 2007; Wang et al., 2014; Sabbah et al., 2016). However, it remains a matter of debate which specific region within the occipital cortex develops stronger functional connectivity with frontal language areas (Noppeney, 2003; Bedny et al., 2011; Watkins et al., 2012; Butt et al., 2013; Striem-Amit et al., 2015). Some studies have reported that V1 has increased functional connectivity with language areas- the inferior frontal 
cortex (IFC) (Butt et al., 2013; Striem-Amit et al., 2015)—in blind individuals relative to sighted controls, while others found that instead of V1, higher-tier 'visual' cortexthe LOC - in blind humans exhibits an enhanced connectivity with the IFC (Noppeney, 2003; Bedny et al., 2011; Watkins et al., 2012). We hypothesized that the LOC in blind individuals may act as the multisensory integration area, bridging tactile Braille recognition and language-related components of Braille reading and that the functional connectivity between the LOC and IFC might support the Braille reading behaviour of blind individuals.

In addition, why the 'visual' cortex engages more actively in various crossmodal tasks in blind humans, including late blind humans, than in sighted controls is still debated (Röder, Kekunnaya and Guerreiro, 2021). Here, we use Braille reading as a model to investigate the hypothesis that 'visual' cortex activity in late blindness predominantly arises from the top-down connectivity from higher-order multisensory or supramodal cortex (Collignon et al., 2013; Röder, Kekunnaya and Guerreiro, 2021). Many researchers have been concerned about the direction of the functional connectivity between the 'visual' and higher-order frontal areas of blind individuals (Bedny et al., 2011; Deen, Saxe and Bedny, 2015; Bedny, 2017). Some researchers have proposed that there might be bidirectional information transmission between these two areas for further information processing (Deen, Saxe and Bedny, 2015). Other researchers have stressed the predominant role of top-down connectivity, e.g., from the frontal cortex, which mediates the activity of the 'visual' cortex in higher-cognitive tasks in blind humans (Bedny et al., 2011; Bedny, 2017). However, studies investigating the direction of the 'visual'-IFC functional connectivity are lacking. The present study directly addressed this open question by examining the dynamic communication between the 'visual' cortex and frontal language areas in blind humans during natural Braille reading.

This study focused on Braille readers with acquired blindness. To fully investigate the behaviour and neural correlates of natural Braille reading, as shown in Figure 1, we first assessed the blind individuals' Braille reading proficiency with natural Braille 
articles and recorded their self-reported amount of Braille reading practice time for different age periods. We quantified the resting-state functional connectivity with frontal language areas for two 'visual' brain regions, V1 and LOC, in blind humans to determine whether this connectivity could predict the Braille reading performance of blind individuals. Furthermore, these blind subjects were asked to perform a natural Braille reading task during functional magnetic resonance imaging (fMRI) acquisition. We then employed dynamic causal modelling (DCM) to investigate the direction of information flow between the 'visual' and frontal language areas in blind humans. 


\section{Results}

A

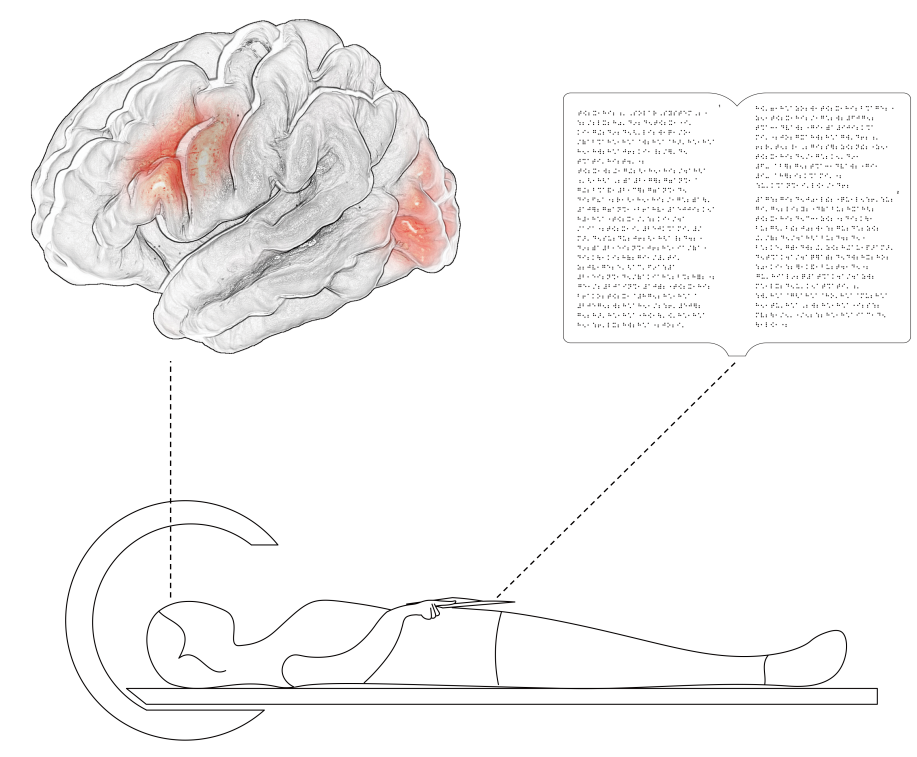

B

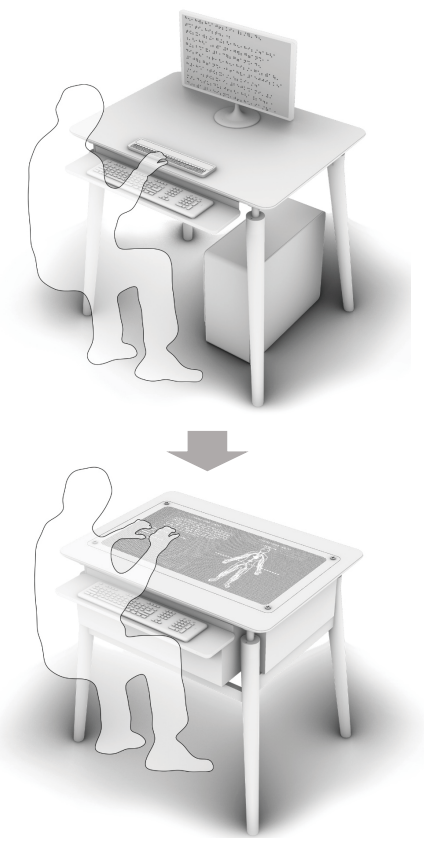

Figure 1 | Schematic overview of the study and natural Braille reading devices for

blind people. (A) Both resting-state signals and dynamic task-related fMRI data were assessed. The resting-state functional connectivity of two visual brain regions (V1 and LOC), especially with the frontal cortex, was compared respectively between acquired blindness and sighted groups. The task fMRI paradigm was designed to simulate reallife Braille reading using large-format, natural Braille texts. (B) Inspiration for the change from a traditional Braille display (upper panel) containing limited, single-line Braille words to the design of a natural Braille reading device (lower panel) enabling continuous Braille article display for blind individuals. 

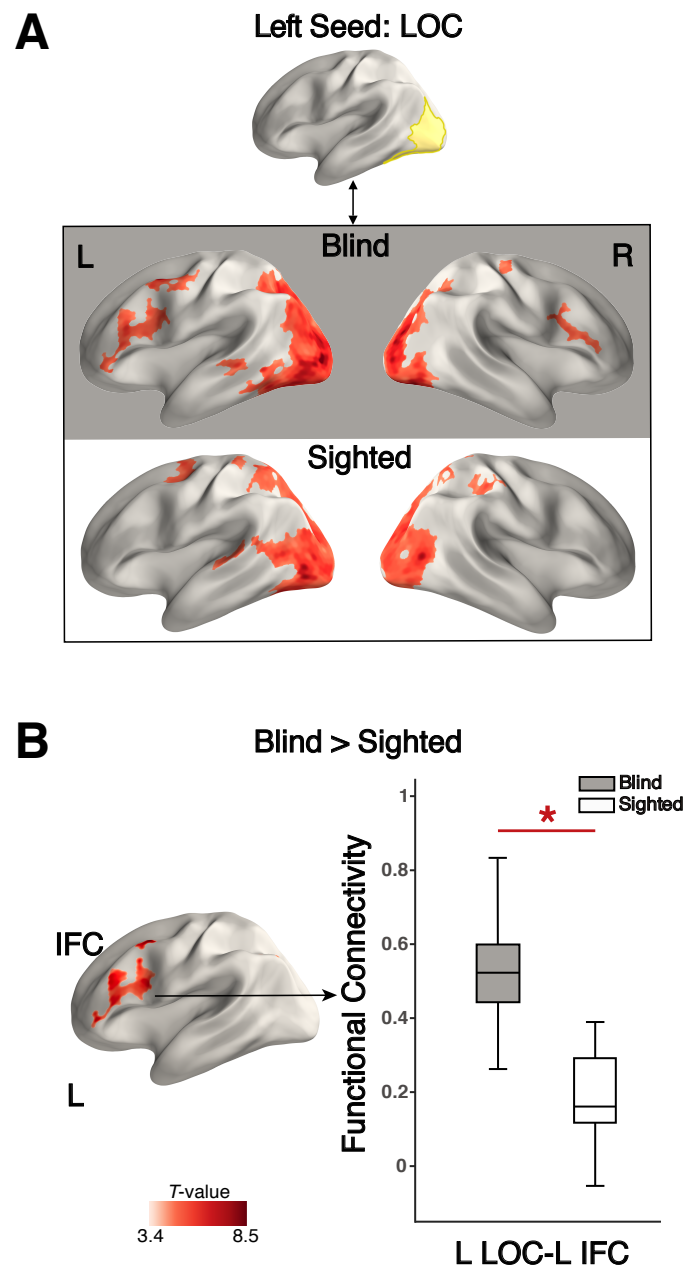
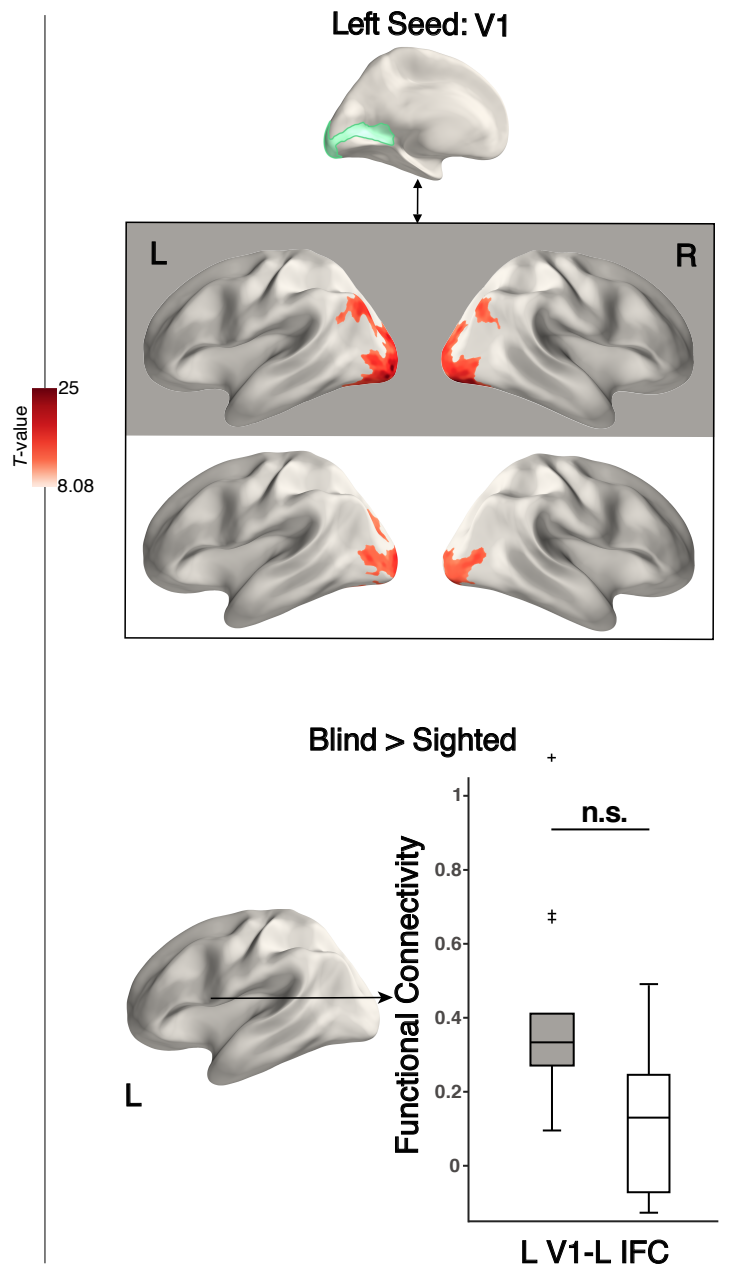

Figure 2 | Functional connectivity patterns for the primary 'visual' cortex (V1) and lateral occipital cortex (LOC). (A) Maps show vertices with significantly positive connectivity with the left LOC seed (vertexwise $P<10^{-6}$, uncorrected, cluster size $>100 \mathrm{~mm}$ ) in the blind group and sighted group (left panel). Maps show vertices with significant positive connectivity with the left V1 seed (vertexwise $P<10^{-6}$, uncorrected, cluster size $>100 \mathrm{~mm}$ ) in the blind group and sighted group (right panel). (B) Differences in functional connectivity patterns for left LOC (left panel) and V1 (right panel) in the blind group versus the sighted group respectively. Significant vertices were corrected for multiple comparisons using permutation testing with threshold-free cluster enhancement $(P<0.025$, corrected for both hemispheres, cluster size $>15 \mathrm{~mm}$ ). Box plot of connectivity strengths (Fisher r-to-z-transformed) between the seed and the left IFC cluster (located from left panel in B) for the two groups. The horizontal line denotes the median, the asterisk indicates significance. V1, primary 
visual cortex (light green); LOC, lateral occipital cortex (light yellow); IFC, inferior frontal cortex. Blind, dark colour; Sighted, light colour. L left hemisphere, R right hemisphere. ${ }^{*} P<0.025$, corrected for both hemispheres. n.s. non-significant.

\section{Distinctive long-range functional connectivity for the higher-tier 'visual' cortex in}

the blind group. Taking the sighted group as the reference, we tested functional connectivity changes in the blind group for two visual brain regions, V1 and LOC, for the two hemispheres separately. The functional connectivity for V1 was not different between the blind and sighted groups. In both groups, we found significant connections of V1 with other visual areas but not with frontal language areas - the IFC (Figure 2, Supplementary Figure 1, right panel). By contrast, we observed significantly strong functional connectivity of the LOC with the left IFC in the blind group rather than in the sighted group (significant difference at $P<0.025$, corrected for both hemispheres; Figure 2, Supplementary Figure 1, left panel). Unsurprisingly, prominent connections of the LOC with other visual regions and extensively with dorsal parietal regions were found in both groups.

In addition, despite significant connections between the LOC seed and IFC in both hemispheres in the blind group $\left(P<10^{-6}\right.$, uncorrected $)$, the functional connectivity strengths of the bilateral LOC with only the left IFC but not the right IFC in the blind group were significantly stronger than those in the sighted controls (thresholded at $P<$ 0.025, corrected for both hemispheres; Figure 2, Supplementary Figure 1, left panel). We also found significantly enhanced functional connectivity between the left LOC and left intraparietal sulcus (IPS) in the blind group than in the sighted group (Supplementary Figure 2). 

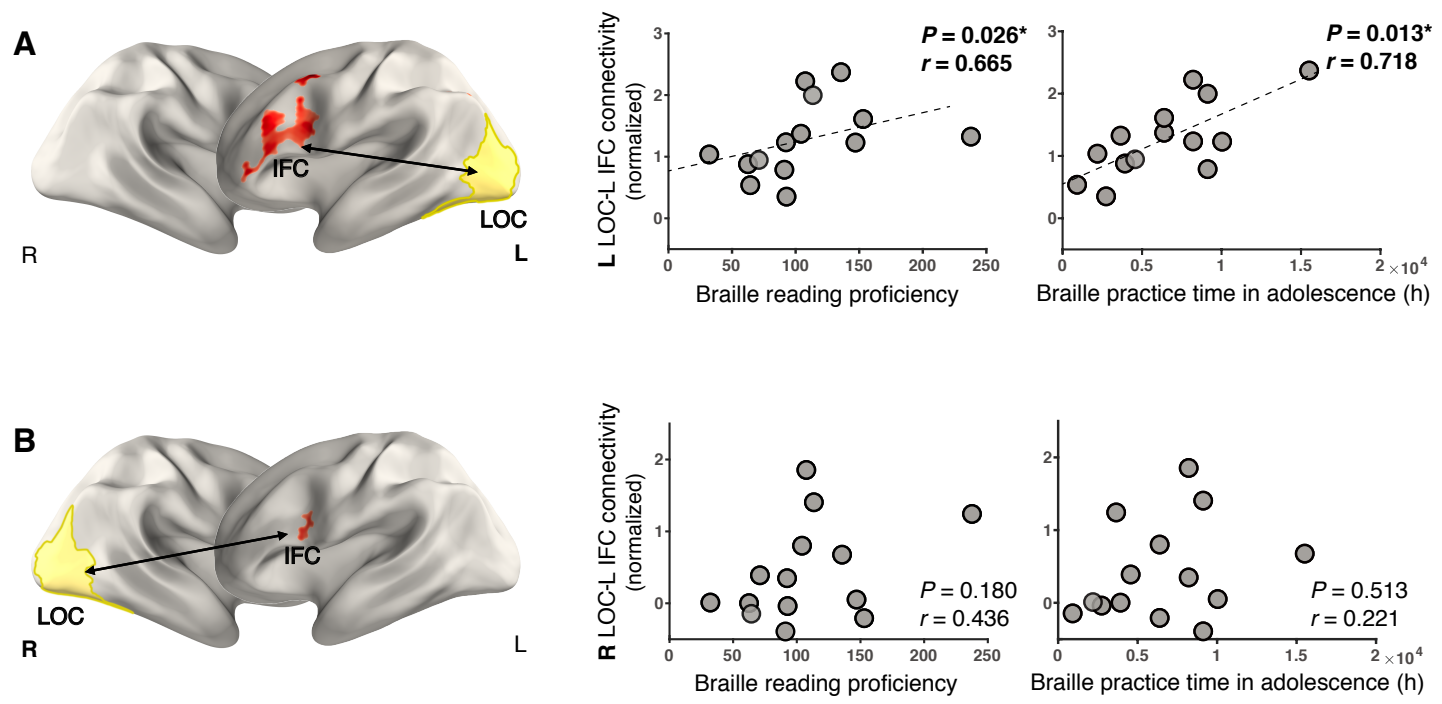

Figure 3 | Left LOC-IFC functional coupling predicts Braille reading proficiency and practice time. (A) ROIs and their connection. Plots of strong and positive correlation between individual strengths of left LOC-IFC connectivity and Braille reading proficiency (left panel) and Braille reading practice time in adolescence (right panel). (B) ROIs and their connection. The plots illustrate that the functional connectivity between the right LOC and left IFC in the blind group had no significant correlation with either Braille reading proficiency (left panel) or Braille reading practice time in adolescence (right panel). L left hemisphere, $\mathrm{R}$ right hemisphere.

\section{Left LOC-IFC functional coupling predicts Braille reading behaviour in blind}

individuals. To test our hypothesis that the reorganized functional connectivity for the LOC is relevant to Braille reading behaviour, we examined whether this functional connectivity predicted individual levels of Braille reading proficiency and whether there was an additional correlation with the amount of Braille reading practice time. We performed a Braille reading behavioural test to estimate the Braille reading proficiency of our blind participants using natural Braille articles. The behavioural tests showed that blind individuals had various levels of Braille reading proficiency, with an average reading speed of $107.52 \pm 50.45$ (mean \pm s.d.) Braille words per minute, and an average accuracy of $99.84 \pm 0.17 \%$ (mean \pm s.d.). We found that a stronger left LOC-IFC functional connectivity contributed to a higher Braille reading proficiency (Spearman correlation, $n=14, P=0.013, r=0.644$; Pearson correlation, $n=14, P=0.159, r=$ 
0.397). This effect remained significant after controlling for the age of blindness onset, age and mean framewise displacement (Power et al., 2012) (motion) in the resting-state scan (Figure 3A left panel, partial Spearman correlation, $P=0.026, r=0.665$; partial Pearson correlation, $P=0.358, r=0.308)$. By contrast, the right LOC-left IFC functional connectivity was not correlated with the blind individual's Braille reading proficiency (Figure 3B left panel, partial Spearman correlation, $P=0.180, r=0.436$; partial Pearson correlation, $P=0.358, r=0.307)$. There was no significant correlation between left V1-IFC connectivity and Braille reading proficiency in the blind group (Supplementary Table 1). The enhanced functional connectivity between the left LOC and IPS in blind individuals was not associated with the Braille reading proficiency, either. (Supplementary Table 1).

Blind participants were further asked to answer a detailed questionnaire on the selfestimated amount of Braille reading practice time for different age periods, including childhood, adolescence, and adulthood. We then calculated the correlations between the functional connectivity for the LOC and individual Braille practice time respectively in each age period. Braille reading practice time (in hours) during adolescence was significantly and positively correlated with the left LOC-IFC functional connectivity in blind individuals (Pearson correlation, $n=14, P=0.003, r=0.724$; Spearman correlation, $n=14, P=0.029, r=0.582)$. This relationship remained significant after controlling for the age of blindness onset, age and head motion (Power et al., 2012) in the scan (Figure 3A right panel, partial Pearson correlation, $P=0.013, r=0.718$; partial Spearman correlation, $P=0.052, r=0.598)$. Additionally, the total Braille reading practice time (in hours) was significantly and positively correlated with the left LOCIFC connectivity (Pearson correlation, $n=14, P=0.032, r=0.574$; Spearman correlation, $n=14, P=0.072, r=0.495)$. It was also nearly significant for the partial correlation (Supplementary Figure 3A). Neither Braille reading practice time during childhood nor adulthood showed a significant correlation with this functional connectivity (Supplementary Figure 3A). By contrast, the right LOC-left IFC functional connectivity was not significantly correlated with blind people's Braille 
reading practice time (in adolescence, Figure 3B right panel, partial Pearson correlation, $P=0.513, r=0.221$; partial Spearman correlation, $P=0.322, r=0.330$; in other age periods, Supplementary Figure 3B). Similarly, the left V1-IFC connectivity and the increased left LOC-IPS connectivity did not correlate with blind individuals' Braille reading practice time (Supplementary Table 1).

In summary, although there was a significant increase in both the left LOC-left IFC and the right LOC-left IFC functional connectivities, only the left-lateralized LOC-IFC functional connectivity was significantly associated with Braille reading behaviours. The left LOC-IFC functional coupling predicted blind individuals' Braille reading proficiency and Braille reading practice time.

A

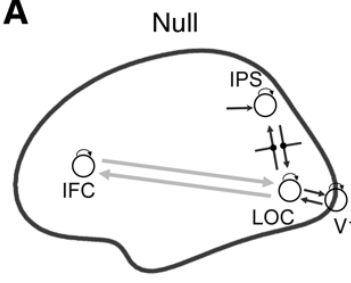

Top-down model

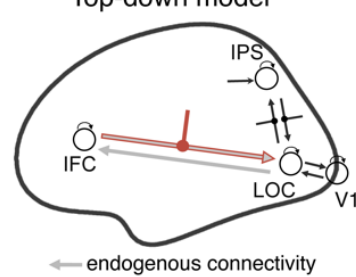

C
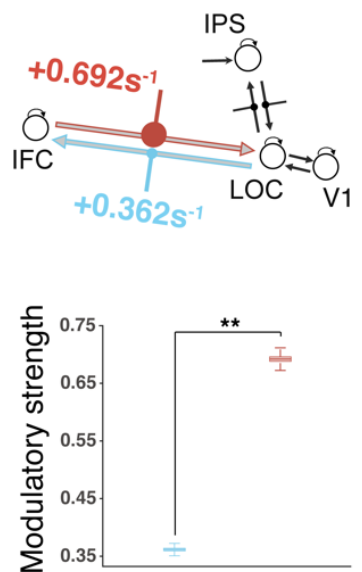
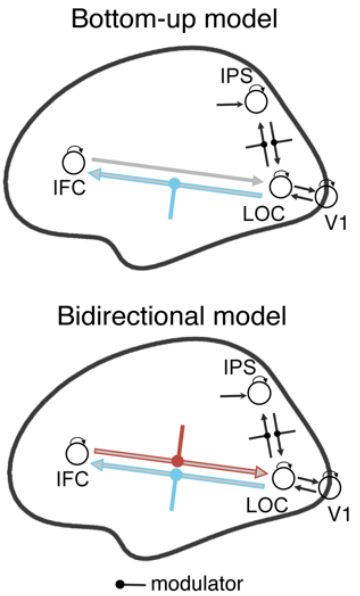

endogenous connectivity

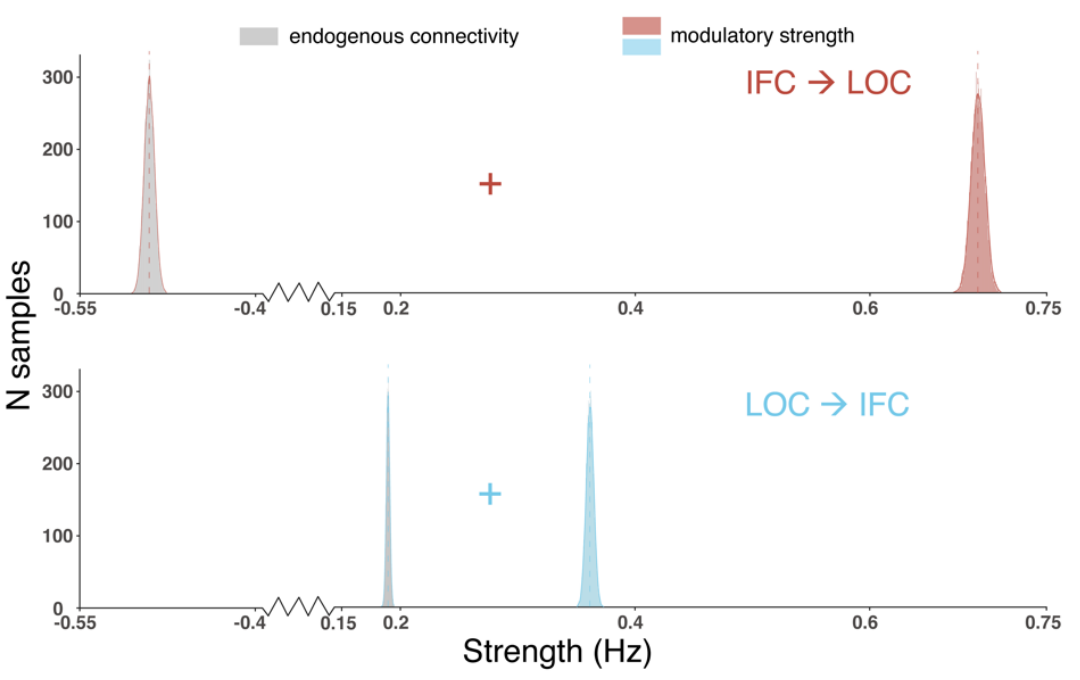

B

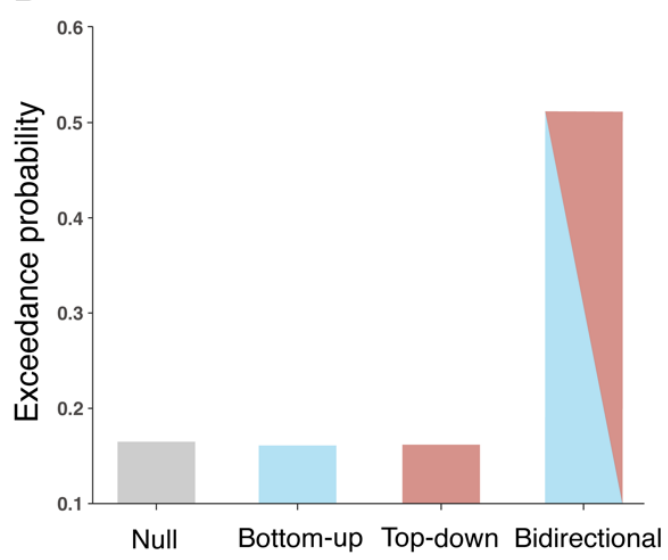

Figure 4 | DCM analyses of left LOC-IFC effective connectivity for natural Braille 
reading. (A) Model space specified by adding task modulation to different directions of the left LOC-IFC connection, resulting in 4 competing models. (B) Exceedance probability for the DCM models, corresponding to the model labels in (B). (C) Illustration of the Bayesian model averaging (BMA) result of the winning bidirectional model for the blind group (all significant at a posterior probability threshold of 0.99 ). Modulators added to the LOC-IFC connection are coded in the size of the filled circles, representing their effect size (left panel). The modulatory strength of Braille reading on the IFC $\rightarrow$ LOC connection was significantly higher than that on the LOC $\rightarrow$ IFC connection $(P<0.001)$. For each parameter (endogenous or modulatory) of the topdown IFC $\rightarrow$ LOC connection and the bottom-up LOC $\rightarrow$ IFC connection, the distribution of the 10,000 samples of the posterior densities is provided respectively. Endogenous connectivity: gray; modulatory strength: red for IFC $\rightarrow$ LOC connection, cyan for LOC $\rightarrow$ IFC connection. $* * P<0.001$.

\section{Bidirectional left LOC-IFC information flow for Braille reading with} asymmetrically stronger top-down modulation. Next, we employed dynamic causal modelling (DCM) to assess the direction of the LOC-IFC effective connectivity during Braille reading and investigated how this connectivity was modulated by the task. The blind subjects were asked to silently read pages containing natural Braille articles while undergoing fMRI, with no-Braille blank pages as the control. The Braille > blank contrast elicited activations in the bilateral V1, LOC, IPS and left IFC in blind subjects (Supplementary Figure 4). In addition, we constrained our DCM models to the left hemisphere due to the left-lateralized activation of the IFC and the left-lateralized increased LOC-IFC and LOC-IPS functional connections in the blind individuals. Therefore, four brain regions, IPS, V1, LOC, and IFC-which are all frequently implicated in Braille reading (Burton, Snyder, Conturo, et al., 2002; Sadato, 2005) were selected for further DCM analysis.

We specified the model space as shown in Figure 4A. The null model represents the basic structure, including endogenous effective connectivity among ROIs and the 
modulation of the IPS-LOC connection confirmed in previous studies (Fujii et al., 2009). Considering the consistent finding that tactile information from the somatosensory cortex was redirected to the occipital cortex through the poly-sensory intraparietal cortex (Wittenberg et al., 2004; Sadato, 2005; Fujii et al., 2009), we used the IPS to model the tactile input for the LOC. To investigate the dynamic interactions between the left LOC and the left IFC in the blind individuals, each direction of this connection can be either modulated or not, thus obtaining four possible models. Individual fixed-effects Bayesian model selection (BMS) showed that the bidirectional modulation model fit the data of most blind individuals best, except for one blind subject, for whom the top-down model was selected. Further application of randomeffects Bayesian model selection (BMS) at the group level showed that the bidirectional model clearly outperformed the other three models (Figure 4B, exceedance probability [the probability that a particular model is more likely than any other model] $=51.24 \%$ ).

Bayesian model averaging (BMA) was applied to the winning model (Supplementary Tables 2,3) and showed that the endogenous connectivity and modulatory effect parameters between the LOC and IFC were all significant (Figure 4C, posterior probabilities $>0.99$ ). As illustrated in Figure 4C, the LOC-to-IFC endogenous connectivity was positive $\left(0.190 \mathrm{~s}^{-1}\right)$, while the IFC-to-LOC connectivity was negative $\left(-0.491 \mathrm{~s}^{-1}\right)$, indicating that irrespective of the task-relevant modulation, the activity changes in the LOC increased the activity in the IFC but not vice versa. The modulation effects of Braille reading on the left LOC-IFC bidirectional connections were both positive in the blind group, with a modulation strength of $+0.362 \mathrm{~s}^{-1}$ for the bottom-up connection and $+0.692 \mathrm{~s}^{-1}$ for the top-down connection (Figure 4C left panel). Notably, the Braille reading task-relevant modulation of the top-down communication from the left IFC to the LOC was significantly stronger than that of the bottom-up communication, from the LOC to the IFC (Figure $4 \mathrm{C}, P<0.001$ ). This asymmetrical modulation highlights the functional relevance to natural Braille reading of the topdown communication from the left IFC to the left LOC in blind individuals. 


\section{Discussion}

In this study, we collected natural Braille reading behaviour data and resting-state and task fMRI data from 14 subjects with acquired blindness, to investigate the behavioural significance of the functional connectivity between the 'visual' cortex and frontal cortex for natural Braille reading, and the neural correlates of the crossmodal plasticity in the 'visual' cortex. We observed that the left higher-tier 'visual' cortex LOC rather than the primary 'visual' cortex V1 developed strong functional connectivity with frontal language area IFC in late blind humans. Importantly, we further showed that the left-lateralized LOC-IFC functional connectivity was positively correlated with the Braille reading practice time of blind individuals, especially in adolescence. And a stronger left LOC-IFC connectivity predicted a higher Braille reading proficiency. In addition, the DCM analysis revealed that increased bidirectional left LOC-IFC information flow with asymmetrically stronger task-relevant top-down modulation emerged in a natural Braille reading task. Our findings thus concur in suggesting that the enhanced functional connectivity between the left LOC and the left IFC in acquired blindness serves as a key neural nexus that bridges tactile Braille recognition and higher-level language processing in natural Braille reading.

The crossmodal recruitment of the typical visual cortex for nonvisual functions in blind humans has been frequently observed (Röder and Neville, 2003; Pavani and Röder, 2012; Renier, De Volder and Rauschecker, 2014; Bedny, 2017; Heimler and Amedi, 2020; Röder, Kekunnaya and Guerreiro, 2021). However, one matter of debate surrounding this dramatic plasticity is whether there is a reversed hierarchy of functional organization across 'visual' areas or not (Amedi et al., 2003; Büchel, 2003; Burton, 2003; Watkins et al., 2012). A study of congenitally blind individuals showed a preference for more 'abstract' aspects (verbal memory) in early V1, while higher-tier visual areas processed more 'sensory' aspects, suggesting a reversal of the normal hierarchy in the visual systems of sighted individuals (Amedi et al., 2003; Büchel, 2003). This is supported by findings of increased long-range connectivity between V1 
and the higher-order frontal cortex in blind humans (Butt et al., 2013; Striem-Amit et al., 2015). Some studies reported activations of both the primary and higher-level 'visual' regions in early and late blind subjects during language-related tasks (Burton, Snyder, Conturo, et al., 2002; Burton, Snyder, Diamond, et al., 2002; Röder et al., 2002; Bedny et al., 2011). Other studies, however, did not support the reversed pattern of organization by specifically identifying the role of the higher-tier 'visual' cortex in cognitive tasks and its functional connectivity with frontal language areas in individuals with early blindness and anophthalmia (Noppeney, 2003; Watkins et al., 2012). The current study showed that late blind individuals had developed increased left LOC-IFC functional connectivity that was positively correlated with their Braille reading performance, instead of the V1-IFC connectivity, which suggested a normal functional hierarchy in 'visual' regions of late blind humans. This is not implausible since late blind individuals might have initially developed their neural circuits as normally sighted people (Röder, Kekunnaya and Guerreiro, 2021). Note that this study employed an anatomically defined higher-tier 'visual' cortex, the LOC, in late blind humans, approximately encompassing the location of the visual word form area (VWFA) in sighted people. The typical VWFA in sighted is a reading-specific area that is tuned to orthographic word structure not only in visual word reading (Cohen and Dehaene, 2004; Dehaene and Cohen, 2011) but also in tactile reading after Braille training (SiudaKrzywicka et al., 2016; Bola et al., 2019; Matuszewski et al., 2021). In tactile Braille reading, similar responses have been found in the 'VWFA' in blind humans to those in the VWFA in sighted humans (Reich et al., 2011; Bedny, 2017). It was suggested that the higher-tier 'visual' cortex served as a particularly important entry node for tactile signals (Sadato, 2005) and extracted abstract Braille shape information for further linguistic-related processing in frontal regions (Sadato, 2005). Additionally, some other studies have reported a less reading-specialized 'VWFA' in blind individuals, and that its activations in some functions such as general language processing (Reich et al., 2011; Kim et al., 2017; Katarzyna Rączy et al., 2019), are similar to those in the higher-tier 'visual' cortex, the LOC (Noppeney, 2003; Bedny et al., 2011; Watkins et al., 2012; Bedny, 2017). With the brain-behaviour evidence in this study, we thus propose that the 
left LOC in late blind humans becomes a more extensive and versatile crossmodal region incorporating a VWFA-like function that bridges tactile Braille orthographic and language processing in Braille reading, resulting in the distinctive neural nexus of the left LOC-IFC. The development of this connectivity might be based on the existing crossmodal connectivity between the left VWFA and the genuine language area, the IFC, in sighted individuals (Behrmann and Plaut, 2013; Yeatman, Rauschecker and Wandell, 2013; Bouhali et al., 2014). This prominent brain rewiring in late blind people also provides new evidence for the "connectivity-based" plasticity theory (Schepers et al., 2012; Bedny, 2017).

How does the observed crossmodal plasticity of the LOC in late blind humans emerge? With DCM analysis, we directly addressed this question by revealing the direction of the information flow on the distinctive neural connection between the left LOC and the higher-order language area, left IFC, during a natural Braille reading task. The winning bidirectional model for the blind subjects (Figure 4A, B) is in line with the Hebbian theory (Hebb, 1950) that the constant information flow between the left LOC and IFC during Braille reading might strengthen such crossmodal connection. Our results thus extend the behavioural principle that "practice makes perfect" by showing positive correlations between this strengthened connectivity in the resting state and both Braille reading practice time and proficiency. In particular, the asymmetrically stronger taskrelevant top-down modulation of the left IFC-to-LOC connection during Braille reading (Figure 4C) provides important evidence for the top-down controlled learning mechanism in late blind humans (Röder, Kekunnaya and Guerreiro, 2021); that is, the crossmodal capacities of the genuine visual cortex in late blind individuals are possibly mediated by feedback connections from higher-order neural systems tuned during taskrelevant learning (Röder, Kekunnaya and Guerreiro, 2021). This is likely essential for late blind individuals because their visual deprivation onset occurs after the pruning of exuberant crossmodal connectivity during the early phases of typical development (Batardière et al., 2002; Röder, Kekunnaya and Guerreiro, 2021); therefore, it might be relatively difficult for them to extract crossmodal characteristics exclusively via 
bottom-up, stimulus-driven input (Rohlf et al., 2017; Röder, Kekunnaya and Guerreiro, 2021). Our finding of the task-specific top-down influence on left LOC activity is in accord with a study that showed stronger task-induced 'visual' cortex activity in individuals with acquired blindness due to active task interpretation (Murphy et al., 2016) as well. This top-down mediated plasticity in late blind humans is compatible with the typical adult neural learning mechanisms demonstrated by several human and non-human animal studies (Keuroghlian and Knudsen, 2007; Bavelier et al., 2010; Rohlf et al., 2017; Röder, Kekunnaya and Guerreiro, 2021). Additionally, as suggested by researchers (Batardière et al., 2002; Magrou et al., 2018; Röder, Kekunnaya and Guerreiro, 2021) that the elaboration of feedback connectivity might require stimulusdriven input, our results further implied that in Braille reading, the bottom-up left LOCIFC effective connectivity provided Braille shape information input to the IFC; thus, the genuine language area, left IFC, predicted the pattern of activity within the 'visual' area, left LOC, based on the prior association of tactile Braille characteristics with semantic and conceptual knowledge (Price and Devlin, 2011) and then delivered topdown modulation to the LOC. This is also in line with the predictive coding framework (Friston, 2003, 2010). Altogether, the present study suggests that the activity of the left LOC in late blind humans does not exclusively arise from sensory input but is predominantly driven by task-relevant top-down information flow from the IFC.

Different from other studies using Braille letters or words, the large-scale Braille articles used in our study made it possible to observe task-relevant top-down communication in blind humans during natural Braille reading. We thus speculate that a promising strategy for blind Braille readers to make better use of this top-down connectivity during natural Braille reading might be to make the context more available. Compared with traditional refreshable Braille displays offering only a single-line of characters (Ren et al., 2008), an emerging technology is the large-print Braille computer (Völkel, Weber and Baumann, 2008; Jiao, Lu and Xu, 2018), which displays continuous and large-scale natural Braille contexts. This new type of Braille reading device likely allows for active understanding and prediction based on contextual information during 
natural Braille reading and may accelerate the purposeful brain rewiring of late blind people.

\section{Methods}

Subjects. 14 blind ( 7 males) and 15 sighted subjects ( 8 males) participated in the study. All subjects were right handed and had no history of neurological disorder. Participants in the blind group were undergraduate students studying at a special education college (mean age \pm s.d. $=21.92 \pm 1.97$ years) and were all Braille readers with acquired blindness (Table 1). Sighted subjects had normal or corrected-to-normal vision and had never learned Braille. One sighted subject with excessive head motion (mean framewise displacement [FD (Power et al., 2012); > 2 SD from the group mean]) during fMRI acquisition was excluded from further analyses, leaving 14 sighted subjects (7 males, mean age \pm s.d. $=21.73 \pm 1.07$ years). The two groups were matched in age (two sample $T$-test, $n=14$ in the blind group and $n=14$ in the sighted group, $T$-value $=0.325$, CI [$1.0541 .443], \mathrm{DF}=26, P=0.748$, Cohen's $d=0.123$, two-tailed). The study was approved by the Institutional Review Board of Tsinghua University, and informed consent was obtained from each of the participants.

Acquisition of biographical data on Braille reading practices. Each blind subject was asked to answer a detailed questionnaire on the self-estimated amount of time (in hours) spent in Braille reading practice during different age periods (Liu et al., 2007). The subjects were asked to retrospectively identify key events in their Braille practice, such as when they started to learn Braille reading and when they changed the amount of time for Braille reading practice. They thereafter estimated the average hours of Braille reading practice per week in the time periods between these key events. We then calculated the total time of Braille reading practice during three different age periods: childhood (from the start of Braille reading to the age of 11 years), adolescence (12-16 years) and adulthood (from the age of 17 years to the exact time of the experiment). See Table 1 for details of the Braille reading practice of the blind subjects. 
Braille reading performance test. The blind subjects were asked to participate in a Braille reading behavioural test resembling real-life reading prior to fMRI acquisition to estimate their Braille reading proficiency. Previous Braille reading behavioural studies have indicated that passage length and content validity may influence the reliability and validity of the assessment (Mason, 2012). The content validity concerns the readability estimates according to the level of difficulty for subjects to read such materials. Given that all of our blind subjects had passed the special college entrance examination for blind individuals, we selected 2 natural Braille articles at the high school level, which was immediately below their current level of Braille reading without understanding difficulties. The average length of each natural Braille article was 500 Braille words. All reading materials were proofread by an experienced Braille reader and were printed by a Braille printer from VIEWPLUS (Viewplus.com) on Braille paper, measuring $27.94 \mathrm{~cm}$ in length and $24.89 \mathrm{~cm}$ in width.

The task was to read the 2 natural Braille articles aloud as accurately and quickly as possible. The reading strategy was not specified. We measured the number of Braille words correctly read per minute on average for each blind subject. If a word was read inaccurately, it was not counted in the final Braille proficiency score. Individual Braille reading accuracies were also assessed by calculating the proportion of Braille words correctly read to the total words in the 2 Braille articles. To avoid situations in which the difficulty in Braille reading comprehension was because of one's unfamiliarity with individual Braille words, blind individuals with a reading accuracy exceeding a threshold of $99.5 \%$ were recruited in this study.

Functional MRI parameters. All MR images were acquired from a Philip Achieva 3.0 Tesla TX MR scanner with a 32-channel head coil. The participants laid supine with their heads snugly fixed with straps and foam pads to minimize head movement. Structural images were acquired using a sagittal magnetization-prepared rapid gradientecho $\mathrm{T} 1$-weighted sequence $\left(\mathrm{TR}=7.6 \mathrm{~ms}, \mathrm{TE}=3.7 \mathrm{~ms}\right.$, flip angle $=8^{\circ}$, voxel size $=1$ $\times 1 \times 1 \mathrm{~mm}, \mathrm{FOV}=256$, slices $=180)$. Before the task session, participants underwent 
two resting-state fMRI scans (8 min per scan) using an echo-planar imaging sequence $\left(\mathrm{TR}=2000 \mathrm{~ms}, \mathrm{TE}=30 \mathrm{~ms}\right.$, flip angle $=90^{\circ}$, voxel size $=3.5 \times 3.5 \times 3.5 \mathrm{~mm}, \mathrm{FOV}=$ 224 , slices $=35$ ). During the resting-state fMRI scan, the participants were instructed to stay awake. Functional images for the natural Braille reading task were also acquired using an echo-planar imaging sequence $\left(\mathrm{TR}=3000 \mathrm{~ms}, \mathrm{TE}=35 \mathrm{~ms}\right.$, flip angle $=90^{\circ}$, voxel size $=3 \times 3 \times 3 \mathrm{~mm}, \mathrm{FOV}=220$, slices $=47$ ). All subjects were asked to keep their eyes closed during the scans.

Resting-state fMRI data preprocessing and analysis. Structural data were processed and reconstructed to individual cortical surface using FreeSurfer. Individual restingstate data were preprocessed using the following procedures: (i) discarding of the first 4 volumes; (ii) slice-timing correction; (iii) rigid body correction for head motion with the AFNI package (Cox, 1996); (iv) functional-anatomical image co-registration; (v) bandpass temporal filtering (0.01-0.08 Hz); and (vi) regressing out of nuisance covariates to control for physiological effects and head motion (6 head motion parameters and 2 regressors corresponding to the ventricular signal and white matter signal). The preprocessed fMRI data of each subject were first aligned to the reconstructed individual cortical surface in each hemisphere. Then, a 6-mm full-width half-maximum (FWHM) Gaussian smoothing kernel was applied to the fMRI data in the surface space. The smoothed data were then down-sampled to the FreeSurfer template with 2,562 vertices in each hemisphere using the mri_surf2surf function in FreeSurfer. To ensure reliable measures of functional connectivity, we combined the fMRI data of two resting-state scans for further analyses.

The primary visual cortex (V1) was defined as the combination of the occipital pole and calcarine sulcus in FreeSurfer's automatic anatomical parcellation (aparc2009) (Destrieux et al., 2010), consistent with the location of V1 in previous studies (Bedny et al., 2011; Watkins et al., 2012; Butt et al., 2013). In agreement with the seed regions in prior functional connectivity analyses of blind and sighted individuals (Bedny et al., 2011; Watkins et al., 2012; Abboud and Cohen, 2019), we used the lateral occipital 
cortex as a higher-tier visual seed, located in the anatomical occipital areas in FreeSurfer aparc2009 atlas (Destrieux et al., 2010) (combining the middle occipital gyrus and sulcus, anterior occipital sulcus, inferior occipital gyrus and sulcus, and lateral occipital-temporal sulcus). The V1 and LOC regions were used as the seeds for functional connectivity (FC) analyses.

To obtain the whole-brain functional connectivity patterns of each seed region, we computed the correlation between the mean blood oxygen level-dependent (BOLD) signal of the seed region and the time series of every other vertex in both hemispheres for each subject. These $r$-maps were next converted into $z$-maps using Fisher's $r$-to- $z$ transformation. All statistical group-level FC pattern analyses were carried out using the Surface-Based Data Processing \& Analysis for (Resting-State) Brain Imaging software (DPABISurf 1.1) (Yan et al., 2016). A one-sample $T$-test ( $n=14$ in the blind group and $n=14$ in the sighted group, $\mathrm{DF}=13$ for each group respectively, one-tailed) was performed for individual FC $z$-maps to generate significant and positive FC maps (vertexwise $P<10^{-6}$, uncorrected, cluster size $>100 \mathrm{~mm}$ ) of the V1 or LOC region for each group. Thus, a union mask including identified significant vertices in either the blind or sighted group was created for further FC pattern comparison between the two groups (Wang et al., 2015). Recent works have consistently suggested that the influence of head motion on intrinsic functional connectivity in the analysis of between-group functional connectivity comparison needs to be further scrutinized regardless of the correction one employs at the individual level (Power et al., 2012; Yan et al., 2013). We therefore calculated the mean framewise displacement (FD) (Power et al., 2012), describing the mean absolute displacement of each brain volume relative to the previous volume in translation and rotation in the $\mathrm{x}, \mathrm{y}$, and $\mathrm{z}$ directions during the resting-state scan for each subject.

With mean FD as the covariate controlling for the head motion effect (Wang et al., 2015), we conducted a two-sample $T$-test ( $n=14$ in blind group and $n=14$ in sighted group, $\mathrm{DF}=26$, two-tailed) on the basis of individual $\mathrm{FC} z$-maps within the union mask 
to examine how the V1 or LOC FC patterns of the blind group changed with respect to the sighted group, and the significant vertices were those surviving the multiple comparison correction using non-parametric permutation testing (Winkler et al., 2016) (5,000 permutations) and threshold-free cluster enhancement (Chen, Lu and Yan, 2018) $(P<0.025$, corrected for both hemispheres, cluster size $>15 \mathrm{~mm})$.

\section{Statistical analysis on the relationship between functional connectivity and}

behavioural data. We further tested for a relationship between enhanced functional connectivity and Braille reading behavioural data in blind individuals. Considering that the overall whole-brain connection strength varies among individuals, we z-normalized the functional connectivity for each subject. We next calculated the Pearson correlation and Spearman correlation coefficients between individuals' normalized 'visual'-related functional connectivity and, separately, their Braille reading proficiency, Braille reading practice time within different age periods and total Braille reading practice time. Moreover, we tested partial correlations among these parameters with covariates of the onset age of blindness, the age when the blind individuals participated in the experiment and motion in the resting state scan indexed by mean FD (Jung et al., 2018).

FMRI task paradigm. A natural Braille reading task with 10 blocks was used in this study (Supplementary Figure 4). In each block, blind subjects were instructed to silently read the Braille article page and the next page with the same material but no Braille content (control: blank) using the right index finger within $20 \mathrm{~s}$ at a constant speed, responding to the audio cue of "Begin" or "Turn the page" played in an earphone. The blind individuals were asked to fully read and comprehend the Braille article. All subjects were instructed to carefully touch and perceive the blank page line by line with the same individual state as in the Braille reading condition. Each audio cue lasted for 1 s. The natural Braille articles were used to engage neural processes underlying approximately real-life Braille reading conditions. Each Braille page had 25 rows; the blank page was printed with the same kind and size of paper as the Braille page. All experimental materials were bound together such that one Braille page was followed 
by one blank page.

Task fMRI Data preprocessing and analysis. The task fMRI data were preprocessed using the FsFast software package (http://surfer.nmr.mgh.harvard.edu/fswiki/FsFast). The preprocessing procedures first included motion correction, slice timing correction, and boundary-based functional-anatomical alignment. Similar to the resting-state fMRI, the task fMRI data of each subject were registered to the reconstructed individual cortical surface. The fMRI data were smoothed with a Gaussian filter (FWHM $=6 \mathrm{~mm}$ ) in the surface space. The smoothed data were then down-sampled to the FreeSurfer template with 2,562 vertices in each hemisphere. One blind subject (B7) who presented with excessive head motion (mean FD (Power et al., 2012); > 2 SD from the group mean) during the Braille reading task fMRI scan was excluded from further GLM and DCM analyses, leaving 13 blind subjects (7 males).

Functional MRI analyses were carried out using Statistical Parametric Mapping toolbox (SPM12, Welcome Department of Imaging Neuroscience, Institute of Neurology, London). For the first-level individual analysis, functional images were modelled with 3 regressors (one for the Braille condition, one for the blank condition, and another for the turning page condition, which was discarded in the analysis) using the general linear model (GLM) method. Head motion regression was also performed in the analysis. We defined a Braille $>$ blank contrast to reveal activated brain regions (significant $P<0.025$ for each hemisphere, uncorrected). We did not set an extremely strict threshold here because the aim of these GLM analyses was to yield brain regions showing taskrelevant effects for further DCM analyses. For group-level GLM analysis, all the blind group subjects' data were concatenated and then analysed by a one-sample $T$-test $(n=$ 13 in the blind group, $\mathrm{DF}=12$, one-tailed). Significant brain activations in the blind group were also revealed by the Braille > blank contrast (Supplementary Figure 4, significant $P<0.025$ for each hemisphere, uncorrected).

Dynamic causal modelling (DCM). All DCM analyses were carried out using SPM12. 
DCM is a computational framework for explaining the effective connectivity and interactions among brain regions that show experimental effects (Friston, Harrison and Penny, 2003). Posterior probability analysis in DCM determines the most likely coupling parameters at the neuronal level given the measured BOLD signals according to the specified model and priors on the coupling and haemodynamic parameters. In our case, we focused on the dynamic interactions among the IPS, LOC, V1 and IFC in the left hemisphere that were activated when the blind subjects were reading the natural Braille articles. The very exact left V1, LOC and IFC areas in the resting-state analysis and the larger anatomical region of the IPS (intraparietal sulcus, defined in the FreeSurfer aparc2009 atlas (Destrieux et al., 2010)) were used as anatomical masks for defining regions of interests (ROIs) later. Each subject-specific ROI consisted of all significant vertices revealed by the Braille $>$ blank contrast exceeding a threshold of $P$ $<0.025$ for each hemisphere (uncorrected, cluster size $>5$ vertices) within the corresponding anatomical mask. The time series of these ROIs were then extracted for each subject. We did not set the threshold of cluster size for one blind subject who lacked a strong response in one ROI to ensure that significant vertices could be identified from each of the 4 ROIs (Zeidman et al., 2019).

Typically, there are 3 types of DCM parameters (Friston, Harrison and Penny, 2003): 1) endogenous parameters, reflecting the task-independent effective connectivity among regions; 2) driving effects, which quantify how neural responses change due to the task stimuli; and 3) modulatory strengths, the parameters of greatest interest, which account for the changes in specific effective connectivity due to the task-relevant conditions. Bidirectional endogenous connections among the 4 ROIs were constructed based on anatomical knowledge and our resting-state functional connectivity results. We used the IPS to model the tactile input for the LOC, consistent with the finding that tactile information from the somatosensory cortex was redirected to the 'visual' systems through the poly-sensory intraparietal cortex in blind people (Wittenberg et al., 2004; Sadato, 2005; Fujii et al., 2009). We set the modulatory effect of Braille reading on the connectivity between the left IPS and LOC in accord with a prior study (Fujii et al., 
2009). We then investigated the task-specific modulatory effect of Braille reading on the connection between the left LOC and IFC. Therefore, we considered that the taskrelevant information flow on any direction (bottom-up and top-down) between these two regions might be modulated, resulting in 4 competing models (Figure 4A).

We first performed Bayesian model selection (BMS) with fixed-effects analysis to select the winning model for each subject and then performed BMS with randomeffects analysis to select the winning model at the group level after balancing the explanation of data and the model complexity. The exceedance probability intuitively presented the relative preference within all models (Figure 4B). To make inferences on the group-level model parameters, Bayesian model averaging (BMA) was then employed on the winning model. BMA can estimate the full posterior density on parameters (Penny et al., 2010). Therefore, the significance of each connectivity parameter could be assessed by the fraction of samples in the posterior density (out of 10,000 sampled data points) that were different from zero (Seghier et al., 2011). The significance of the difference in modulatory strengths on one connection versus another connection was assessed by the fraction of samples that were higher for one connection than another (Seghier et al., 2011).

Data availability. The data supporting the findings of this study are available from the corresponding author upon reasonable request.

Code availability. The code used in this article will be available upon publication. 


\section{References}

Abboud, S. and Cohen, L. (2019) 'Distinctive Interaction Between Cognitive Networks and the Visual Cortex in Early Blind Individuals', Cerebral cortex (New York, N.Y. : 1991), 29(11), pp. 4725-4742. doi: 10.1093/cercor/bhz006.

Amedi, A. et al. (2003) 'Early "visual” cortex activation correlates with superior verbal memory performance in the blind', Nature Neuroscience, 6(7), pp. 758-766. doi: $10.1038 / \mathrm{nn} 1072$.

Batardière, A. et al. (2002) 'Early specification of the hierarchical organization of visual cortical areas in the macaque monkey', Cerebral Cortex, 12(5), pp. 453-465. doi: $10.1093 /$ cercor/12.5.453.

Bavelier, D. et al. (2010) 'Removing brakes on adult brain plasticity: From molecular to behavioral interventions', Journal of Neuroscience, 30(45), pp. 14964-14971. doi: 10.1523/JNEUROSCI.4812-10.2010.

Bedny, M. et al. (2011) 'Language processing in the occipital cortex of congenitally blind adults', Proceedings of the National Academy of Sciences. doi: 10.1073/pnas.1014818108.

Bedny, M. (2017) 'Evidence from Blindness for a Cognitively Pluripotent Cortex', Trends in Cognitive Sciences. Elsevier Ltd, 21(9), pp. 637-648. doi: 10.1016/j.tics.2017.06.003.

Behrmann, M. and Plaut, D. C. (2013) 'Distributed circuits, not circumscribed centers, mediate visual recognition', Trends in Cognitive Sciences. Elsevier Ltd, 17(5), pp. 210-219. doi: 10.1016/j.tics.2013.03.007.

Bola, Ł. et al. (2019) 'Functional hierarchy for tactile processing in the visual cortex of sighted adults', NeuroImage, 202(July). doi: 10.1016/j.neuroimage.2019.116084. Bouhali, F. et al. (2014) 'Anatomical connections of the visual word form area', Journal of Neuroscience, pp. 15402-15414. doi: 10.1523/JNEUROSCI.491813.2014 .

Büchel, C. (2003) 'Cortical hierarchy turned on its head', Nature Neuroscience, 6(7), pp. 657-658. doi: 10.1038/nn0703-657.

Burton, H., Snyder, A. Z., Conturo, T. E., et al. (2002) 'Adaptive Changes in Early 
and Late Blind: A fMRI Study of Braille Reading', Journal of Neurophysiology, 87(6), pp. 589-607. doi: 10.1152/jn.00129.2002.

Burton, H., Snyder, A. Z., Diamond, J. B., et al. (2002) 'Adaptive changes in early and late blind: A fMRI study of verb generation to heard nouns', Journal of Neurophysiology, 88(6), pp. 3359-3371. doi: 10.1152/jn.00129.2002.

Burton, H. (2003) 'Visual cortex activity in early and late blind people', Journal of Neuroscience, 23(10), pp. 4005-4011.

Butt, O. H. et al. (2013) 'The fine-scale functional correlation of striate cortex in sighted and blind people', Journal of Neuroscience, 33(41), pp. 16209-16219. doi: 10.1523/JNEUROSCI.0363-13.2013.

Chen, X., Lu, B. and Yan, C. G. (2018) 'Reproducibility of R-fMRI metrics on the impact of different strategies for multiple comparison correction and sample sizes', Human Brain Mapping, 39(1), pp. 300-318. doi: 10.1002/hbm.23843.

Cohen, L. and Dehaene, S. (2004) 'Specialization within the ventral stream: The case for the visual word form area', NeuroImage, 22(1), pp. 466-476. doi:

10.1016/j.neuroimage.2003.12.049.

Cohen, L. G. et al. (1997) 'Functional relevance of cross-modal plasticity in blind humans', Nature, 389(28), pp. 180-183. Available at:

http://neurosciences.us/courses/systems/CentralPlas/cohen97.pdf.

Collignon, O. et al. (2013) 'Impact of blindness onset on the functional organization and the connectivity of the occipital cortex', Brain, 136(9), pp. 2769-2783. doi: 10.1093/brain/awt176.

Cox, R. W. (1996) 'AFNI: Software for analysis and visualization of functional magnetic resonance neuroimages', Computers and Biomedical Research, 29(3), pp. 162-173. doi: 10.1006/cbmr.1996.0014.

Deen, B., Saxe, R. and Bedny, M. (2015) 'Occipital Cortex of Blind Individuals Is Functionally Coupled with Executive Control Areas of Frontal Cortex', Journal of Cognitive Neuroscience, 27(8), pp. 1633-1647. doi: 10.1162/jocn.

Dehaene, S. and Cohen, L. (2011) 'The unique role of the visual word form area in reading', Trends in Cognitive Sciences, 15(6), pp. 254-262. doi: 
10.1016/j.tics.2011.04.003.

Destrieux, C. et al. (2010) 'Automatic parcellation of human cortical gyri and sulci using standard anatomical nomenclature', NeuroImage. Elsevier Inc., 53(1), pp. 1-15. doi: 10.1016/j.neuroimage.2010.06.010.

Friston, K. (2003) 'Learning and inference in the brain', Neural Networks, 16(9), pp. 1325-1352. doi: 10.1016/j.neunet.2003.06.005.

Friston, K. (2010) 'The free-energy principle: A unified brain theory?', Nature Reviews Neuroscience. Nature Publishing Group, 11(2), pp. 127-138. doi: $10.1038 / \mathrm{nrn} 2787$.

Friston, K. J., Harrison, L. and Penny, W. (2003) 'Dynamic causal modelling', NeuroImage, 19(4), pp. 1273-1302. doi: 10.1016/S1053-8119(03)00202-7. Fujii, T. et al. (2009) 'An investigation of cross-modal plasticity of effective connectivity in the blind by dynamic causal modeling of functional MRI data', Neuroscience Research, 65(2), pp. 175-186. doi: 10.1016/j.neures.2009.06.014. Hamilton, R. et al. (2000) 'Alexia for Braille following bilateral occipital stroke in an early blind woman', NeuroReport, 11(2), pp. 237-240. doi: 10.1097/00001756200002070-00003.

Hebb, D. O. (1950) 'The Organization of Behavior; A Neuropsychological Theory', The American Journal of Psychology, 63(4), p. 633. doi: 10.2307/1418888.

Heimler, B. and Amedi, A. (2020) 'Are critical periods reversible in the adult brain? Insights on cortical specializations based on sensory deprivation studies', Neuroscience and Biobehavioral Reviews. Elsevier, 116(June 2019), pp. 494-507. doi: 10.1016/j.neubiorev.2020.06.034.

Jiao, Y., Lu, X. and Xu, Y. (2018) 'A graphical tactile display for the visually impaired', p. 2018.

Jung, W. H. et al. (2018) 'Amygdala Functional and Structural Connectivity Predicts Individual Risk Tolerance', Neuron. Elsevier Inc., 98(2), pp. 394-404.e4. doi: 10.1016/j.neuron.2018.03.019.

Katarzyna Rączy et al. (2019) 'Orthographic Priming in Braille Reading as Evidence for Task-specific Reorganization in the Ventral Visual Cortex of the Congenitally 
Blind', Journal of Cognitive Neuroscience, 31(7), pp. 1065-1078. doi: 10.1162/jocn. Keuroghlian, A. S. and Knudsen, E. I. (2007) 'Adaptive auditory plasticity in developing and adult animals', Progress in Neurobiology, 82(3), pp. 109-121. doi: 10.1016/j.pneurobio.2007.03.005.

Kim, J. S. et al. (2017) 'Development of the Visual Word Form Area Requires Visual Experience: Evidence from Blind Braille Readers', The Journal of Neuroscience, 37(47), pp. 11495-11504. doi: 10.1523/jneurosci.0997-17.2017.

Liu, Y. et al. (2007) 'Whole brain functional connectivity in the early blind', Brain, pp. 2085-2096. doi: 10.1093/brain/awm121.

Magrou, L. et al. (2018) 'How areal specification shapes the local and interareal circuits in a macaque model of congenital blindness', Cerebral Cortex, 28(8), pp. 3017-3034. doi: 10.1093/cercor/bhy125.

Mason, L. K. (2012) Experimental investigation of hand and finger usage in braille reading. University of Northern Colorado. Available at: https://digscholarship.unco.edu/dissertations/209.

Matuszewski, J. et al. (2021) 'Brain plasticity dynamics during tactile Braille learning in sighted subjects: Multi-contrast MRI approach', NeuroImage, 227(December 2020), p. 117613. doi: 10.1016/j.neuroimage.2020.117613.

Murphy, M. C. et al. (2016) 'Top-down influence on the visual cortex of the blind during sensory substitution', NeuroImage. Elsevier Inc., 125, pp. 932-940. doi: 10.1016/j.neuroimage.2015.11.021.

Noppeney, U. (2003) 'Effects of visual deprivation on the organization of the semantic system', Brain, 126(7), pp. 1620-1627. doi: 10.1093/brain/awg152. Pascual-Leone, A. et al. (2005) 'the Plastic Human Brain Cortex', Annual Review of Neuroscience, 28(1), pp. 377-401. doi: 10.1146/annurev.neuro.27.070203.144216. Pavani, F. and Röder, B. (2012) 'Crossmodal plasticity as a consequence of sensory loss: Insights from blindness and deafness', in Stein, B. E. (ed.) The new handbook of multisensory processes. Cambridge, MA: MIT Press, pp. 737-760.

Penny, W. D. et al. (2010) 'Comparing families of dynamic causal models', PLoS Computational Biology, 6(3). doi: 10.1371/journal.pcbi.1000709. 
Power, J. D. et al. (2012) 'Spurious but systematic correlations in functional connectivity MRI networks arise from subject motion', NeuroImage. Elsevier Inc., 59(3), pp. 2142-2154. doi: 10.1016/j.neuroimage.2011.10.018.

Price, C. J. and Devlin, J. T. (2011) 'The Interactive Account of ventral occipitotemporal contributions to reading', Trends in Cognitive Sciences. Elsevier Ltd, 15(6), pp. 246-253. doi: 10.1016/j.tics.2011.04.001.

Reich, L. et al. (2011) 'A ventral visual stream reading center independent of visual experience', Current Biology, pp. 363-368. doi: 10.1016/j.cub.2011.01.040.

Ren, K. et al. (2008) 'A compact electroactive polymer actuator suitable for refreshable Braille display', Sensors and Actuators, A: Physical, 143(2), pp. 335-342. doi: 10.1016/j.sna.2007.10.083.

Renier, L., De Volder, A. G. and Rauschecker, J. P. (2014) 'Cortical plasticity and preserved function in early blindness', Neuroscience and Biobehavioral Reviews. Elsevier Ltd, 41, pp. 53-63. doi: 10.1016/j.neubiorev.2013.01.025.

Röder, B. et al. (2002) 'Speech processing activates visual cortex in congenitally blind humans', European Journal of Neuroscience. doi: 10.1046/j.14609568.2002.02147.x.

Röder, B., Kekunnaya, R. and Guerreiro, M. J. S. (2021) 'Neural mechanisms of visual sensitive periods in humans', Neuroscience and Biobehavioral Reviews, 120(July 2020), pp. 86-99. doi: 10.1016/j.neubiorev.2020.10.030.

Röder, B. and Neville, H. J. (2003) 'Developmental plasticity', in Grafman, J. and Robertson, I. (eds) Handbook of Neuropsychology. Elsevier, pp. 231-270.

Rohlf, S. et al. (2017) 'Infants are superior in implicit crossmodal learning and use other learning mechanisms than adults', eLife, 6, pp. 1-23. doi: 10.7554/eLife.28166. Sabbah, N. et al. (2016) 'Increased functional connectivity between language and visually deprived areas in late and partial blindness', NeuroImage, pp. 162-173. doi: 10.1016/j.neuroimage.2016.04.056.

Sadato, N. et al. (1998) 'Neural networks for Braille reading by the blind', Brain, 121(7), pp. 1213-1229. doi: 10.1093/brain/121.7.1213.

Sadato, N. (2005) 'How the blind "see" Braille: Lessons from functional magnetic 
resonance imaging', Neuroscientist, 11(6), pp. 577-582. doi: $10.1177 / 1073858405277314$.

Schepers, I. M. et al. (2012) 'Functionally specific oscillatory activity correlates between visual and auditory cortex in the blind', Brain, 135(3), pp. 922-934. doi: 10.1093/brain/aws014.

Seghier, M. L. et al. (2011) 'Lateralization is predicted by reduced coupling from the left to right prefrontal cortex during semantic decisions on written words', Cerebral Cortex, 21(7), pp. 1519-1531. doi: 10.1093/cercor/bhq203.

Siuda-Krzywicka, K. et al. (2016) 'Massive cortical reorganization in sighted braille readers', eLife, 5(MARCH2016), pp. 1-26. doi: 10.7554/eLife.10762.

Striem-Amit, E. et al. (2015) 'Functional connectivity of visual cortex in the blind follows retinotopic organization principles', Brain, 138(6), pp. 1679-1695. doi: 10.1093/brain/awv083.

Völkel, T., Weber, G. and Baumann, U. (2008) 'Tactile graphics revised: The novel BrailleDis 9000 pin-matrix device with multitouch input', Lecture Notes in Computer Science (including subseries Lecture Notes in Artificial Intelligence and Lecture Notes in Bioinformatics), 5105 LNCS(March 2015), pp. 835-842. doi: 10.1007/9783-540-70540-6_124.

Wang, D. et al. (2014) 'Altered resting-state network connectivity in congenital blind', Human Brain Mapping, 35(6), pp. 2573-2581. doi: 10.1002/hbm.22350. Wang, X. et al. (2015) 'Reading without speech sounds: VWFA and its connectivity in the congenitally deaf', Cerebral Cortex. doi: 10.1093/cercor/bhu044.

Watkins, K. E. et al. (2012) 'Language networks in anophthalmia: Maintained hierarchy of processing in "visual" cortex', Brain, 135(5), pp. 1566-1577. doi: 10.1093/brain/aws067.

Winkler, A. M. et al. (2016) 'Faster permutation inference in brain imaging', NeuroImage. The Authors, 141, pp. 502-516. doi: 10.1016/j.neuroimage.2016.05.068.

Wittenberg, G. F. et al. (2004) 'Functional connectivity between somatosensory and visual cortex in early blind humans', European Journal of Neuroscience, 20(7), pp. 
1923-1927. doi: 10.1111/j.1460-9568.2004.03630.x.

Yan, C. G. et al. (2013) 'A comprehensive assessment of regional variation in the impact of head micromovements on functional connectomics', NeuroImage. Elsevier Inc., 76, pp. 183-201. doi: 10.1016/j.neuroimage.2013.03.004.

Yan, C. G. et al. (2016) 'DPABI: Data Processing \& Analysis for (Resting-State)

Brain Imaging', Neuroinformatics. Neuroinformatics, 14(3), pp. 339-351. doi: $10.1007 / \mathrm{s} 12021-016-9299-4$.

Yeatman, J. D., Rauschecker, A. M. and Wandell, B. A. (2013) 'Anatomy of the visual word form area: adjacent cortical circuits and long-range white matter connections', 71(2), pp. 233-236. doi: 10.1038/mp.2011.182.doi.

Zeidman, P. et al. (2019) 'A guide to group effective connectivity analysis, part 1: First level analysis with DCM for fMRI', NeuroImage, 200(May), pp. 174-190. doi: 10.1016/j.neuroimage.2019.06.031.

Acknowledgements. This work is supported by the National Science Foundation of China [grant number NSFC 61621136008] and National Key R\&D Program of China [grant number 2017YFA0205904 B.H.] We thank Brigitte Röder at the University of Hamburg for comments on the manuscript.

Author contributions. B.H., Y.X., R.W. and J.G. designed the research; R.W. and J.G. performed the research; R.W., J.G. and C.Z. collected the data; R.W. and J.G. analysed the data; R.W., B.H., J.G. and Y.X. wrote and improved the paper.

Competing interests: The authors declare no competing financial interests. 
Table 1 Characteristic of blind subjects.

\begin{tabular}{|c|c|c|c|c|c|c|c|c|c|}
\hline & \multirow[b]{2}{*}{ Sex } & \multirow[b]{2}{*}{ Age } & \multirow{2}{*}{$\begin{array}{c}\text { Onset Age } \\
\text { (years) }\end{array}$} & \multicolumn{4}{|c|}{ Braille reading practice time } & \multirow{2}{*}{$\begin{array}{l}\text { Braille Reading } \\
\text { Proficiency } \\
\text { (words/min) }\end{array}$} & \multirow[b]{2}{*}{ Cause of Blindness } \\
\hline & & & & $\begin{array}{c}\text { Start Age } \\
\text { (years) }\end{array}$ & $\begin{array}{l}\text { Childhood } \\
\text { (h) }\end{array}$ & $\begin{array}{c}\text { Adolescence } \\
\text { (h) }\end{array}$ & $\begin{array}{l}\text { Adulthood } \\
\text { (h) }\end{array}$ & & \\
\hline B1 & $\mathrm{F}$ & 23.54 & 14.72 & 9.88 & 4255.90 & 8212.50 & 1769.21 & 92.41 & Glaucoma \\
\hline $\mathrm{B} 2$ & $\mathrm{M}$ & 21.39 & 4.41 & 7.68 & 3153.60 & 9125.00 & 2951.03 & 91.15 & Ocular pathology \\
\hline B3 & $\mathrm{F}$ & 22.54 & 4.12 & 9.84 & 2759.40 & 2737.50 & 1193.55 & 92.85 & Unknown \\
\hline B4 & $\mathrm{F}$ & 22.00 & 5.41 & 7.29 & 7736.18 & 6387.50 & 1095.00 & 104.19 & Optic nerve atrophy \\
\hline B5 & $\mathrm{M}$ & 19.20 & 12.00 & 6.88 & 2802.00 & 3650.00 & 83.43 & 237.85 & Retinal detachment \\
\hline B6 & $\mathrm{F}$ & 20.31 & 5.41 & 7.99 & 2194.50 & 6387.50 & 449.43 & 153.08 & Trauma \\
\hline B7 & M & 19.73 & 15.00 & 15.00 & - & 2190.00 & 680.50 & 32.10 & Macular discoloration \\
\hline B8 & M & 23.28 & 11.18 & 13.93 & - & 3923.50 & - & 62.51 & Glaucoma \\
\hline B9 & $\mathrm{F}$ & 23.87 & 7.00 & 7.52 & 1637.00 & 912.50 & 205.29 & 64.33 & Optic nerve atrophy \\
\hline B10 & M & 20.35 & 11.00 & 6.99 & 10065.00 & 10037.50 & 339.86 & 147.04 & Unknown \\
\hline B11 & $\mathrm{M}$ & 24.44 & 9.00 & 11.08 & 1002.00 & 8212.50 & 220.14 & 107.57 & Ocular pathology \\
\hline B12 & $\mathrm{F}$ & 18.68 & 6.00 & 7.13 & 6221.43 & 9125.00 & 489.10 & 113.50 & Trauma \\
\hline B13 & $\mathrm{F}$ & 23.46 & 8.00 & 6.91 & 8360.33 & 15512.50 & 2917.39 & 135.68 & Glaucoma \\
\hline B14 & $\mathrm{M}$ & 24.14 & 10.00 & 7.28 & 4307.00 & 4562.50 & 848.89 & 71.07 & Glaucoma \\
\hline
\end{tabular}

